\title{
Besov Characteristic of a Distribution
}

\author{
Béatrice VEDEL \\ Laboratoire d'Analyse et de Mathématiques Appliquéss \\ Université Paris XII \\ Avenue du Général De Gaulle \\ 94010 Créteil Cedex — France \\ beatrice.vedel@u-picardie.fr
}

Received: December 23, 2006

Accepted: March 5, 2007

\begin{abstract}
The Besov characteristic of a distribution $f$ is the function $s_{f}$ defined for $0 \leq t<\infty$ by

$$
s_{f}(t)=\sup \left\{s \in \mathbb{R} ; f \in B_{1 / t, 1}^{s}\left(\mathbb{R}^{n}\right)\right\} .
$$

We give in this paper a criterion for a function $\Gamma$ defined on $[0,+\infty[$ to be the Besov characteristic of a distribution. Generalizations of this criterion to particular weighted Besov spaces and to anisotropic Besov spaces are also given.

Key words: Besov spaces, wavelet analysis, weighted Besov spaces, anisotropic Besov spaces, anisotropic wavelet analysis.

2000 Mathematics Subject Classification: 46E35, 42B35, 42C40.
\end{abstract}

\section{Introduction}

The Besov characteristic of a distribution $f$ is the frontier of its Besov domain, the set of indices $(s, t)$ such that $f$ belongs to $B_{1 / t, 1}^{s}\left(\mathbb{R}^{n}\right)$. To determine the Besov characteristic of a distribution gives us information about its global regularity.

Our purpose in this paper is to give a sufficient and necessary condition under which a curve $s=s(t)$ is the Besov characteristic of a distribution. The proof mainly relies on characterization of Besov spaces in wavelet expansions (using a Meyer basis). We also generalize the criterion to the cases of weighted and anisotropic Besov spaces.

Similar results have been given by Triebel in $[6$, chapter 1 , section 18 , and chapter 7 , section 2] in the case of compactly supported distributions with the following result. 
Theorem. The Besov characteristic $\Gamma$ of a compactly supported distribution $f \in \mathcal{S}^{\prime}\left(\mathbb{R}^{n}\right)$ with non-empty singular support defined by

$$
\Gamma_{f}(t)=\sup \left\{s \in \mathbb{R} ; f \in B_{1 / t, \infty}^{s}\left(\mathbb{R}^{n}\right)\right\}
$$

is a continuous, non-decreasing and concave function of slope bounded by $n$. Conversely, if $\Gamma$ is a continuous, non-decreasing and concave function on $\mathbb{R}^{+}$of slope bounded by $n$, there exists $f \in \mathcal{S}^{\prime}\left(\mathbb{R}^{n}\right)$ with a singular support of measure equal to 0 , such that $\Gamma_{f}=\Gamma$.

The definition of the singular support is recalled in section 1 .

Jaffard in $[2,3]$ in the context of multifractal formalism has also given the following criteria in the local case.

Theorem. The (local) Besov characteristic of a distribution $f \in \mathcal{S}^{\prime}\left(\mathbb{R}^{n}\right)$ defined by

$$
\omega_{f, \text { loc }}(t)=\sup \left\{s \in \mathbb{R} ; f \in B_{1 / t, \infty, l o c}^{s}\left(\mathbb{R}^{n}\right)\right\}
$$

is a continuous, non-decreasing and concave function of slope bounded by $n$. Conversely, if $\Gamma$ is a continuous, non-decreasing and concave function on $\mathbb{R}^{+}$of slope bounded by $n$, there exists $f \in \mathcal{S}^{\prime}\left(\mathbb{R}^{n}\right)$ such that $\Gamma_{f}=\omega_{f, \text { loc }}$.

Without assumptions on the localization of the distribution, we show that the profile of the Besov characteristic can be quite different and more cases appear. In addition, for any admissible curve, we construct explicitly a distribution which has for Besov characteristic this curve. Moreover, this distribution can be chosen with an empty singular support, i.e., it locally belongs to $\mathcal{C}^{\infty}\left(\mathbb{R}^{n}\right)$. It means that the Besov characteristic of the distribution can only depend of its global behavior.

\section{Definition, first properties and main result}

We define a smooth resolution of unity $\varphi_{j}, j \geq 0$, in the following way. The function $\varphi_{0}$ is in $\mathcal{C}^{\infty}\left(\mathbb{R}^{n}\right)$, is supported on the ball $\left\{\xi \in \mathbb{R}^{n},|\xi| \leq 2\right\}$, and is identically equal to 1 on the ball $\left\{\xi \in \mathbb{R}^{n},|\xi| \leq 1\right\}$. For $j \geq 1$, we define $\varphi_{j}$, for $\xi \in \mathbb{R}^{n}$, by

$$
\varphi_{j}(\xi)=\varphi_{0}\left(2^{-j} \xi\right)-\varphi_{0}\left(2^{-j+1} \xi\right) \text {. }
$$

We define the operator $\Delta_{j}, j \in \mathbb{N}_{0}$, on $\mathcal{S}^{\prime}\left(\mathbb{R}^{n}\right)$ by

$$
\widehat{\left(\Delta_{j} f\right)}=\varphi_{j} \hat{f} .
$$

For $0<p \leq \infty, 0<q \leq \infty$, and $s \in \mathbb{R}$, the Besov space $B_{p, q}^{s}\left(\mathbb{R}^{n}\right)$ is defined by

$$
B_{p, q}^{s}\left(\mathbb{R}^{n}\right)=\left\{f \in \mathcal{S}^{\prime}\left(\mathbb{R}^{n}\right) ;\|f\|_{B_{p, q}^{s}}=\left(\sum_{j=0}^{\infty} 2^{j s q}\left\|\Delta_{j}(f)\right\|_{L^{p}}^{q}\right)^{1 / q}<\infty\right\}
$$

(usual modification if $q=\infty$ ). 
Definition 1.1. Let $f \in \mathcal{S}^{\prime}\left(\mathbb{R}^{n}\right)$. The Besov characteristic of $f$ is the function defined on $[0, \infty[$, at values in $\overline{\mathbb{R}}$, by

$$
s_{f}(t)=\sup \left\{s \in \mathbb{R} ; f \in B_{1 / t, 1}^{s}\left(\mathbb{R}^{n}\right)\right\} .
$$

For example, the Besov characteristic of a function $f \in \mathcal{S}\left(\mathbb{R}^{n}\right)$ is $s_{f} \equiv+\infty$.

Remark. We fix in the definition of the Besov characteristic $q=1$. By an immediate rescaling, the results we will give can be obtained for any choice of $q, 0<q \leq \infty$.

Proposition 1.2. Let $f$ be a distribution and $s_{f}$ its Besov characteristic. Then $s_{f}$ satisfies one of the following cases:

(i) $s_{f}=+\infty$ on $[0,+\infty[$.

(ii) $s_{f}=-\infty$ on $] 0,+\infty[$.

(iii) $s_{f}$ is continuous, concave and $s_{f}^{\prime} \leq n$ on $[0, \infty[$.

(iv) There exists $t_{0}>0$ such that

$$
s_{f}(t)= \begin{cases}+\infty & \text { if } t \in\left[0, t_{0}[\right. \\ s_{0} & \text { if } t=t_{0}, s_{0} \in \overline{\mathbb{R}} \\ -\infty & \text { if } t \in] t_{0},+\infty[\end{cases}
$$

(v) There exists $t_{0}>0$ such that

$$
s_{f}(t)= \begin{cases}g(t) & \text { if } t \in\left[0, t_{0}[,\right. \\ s_{0}, & \text { if } t=t_{0}, \quad s_{0} \in \overline{\mathbb{R}}, \quad s_{0} \leq \lim _{t \rightarrow t_{0}^{-}} g(t), \\ -\infty & \text { if } t \in] t_{0},+\infty[\end{cases}
$$

where $g$ is continuous, concave and $g^{\prime} \leq n$ on $\left[0, t_{0}[\right.$.

Proof. Let us recall the following embeddings (which can be found, for example, in $\left[1\right.$, chapter 2 , section 3 , and chapter 4 , section 3.1]). For all $0<p \leq p^{\prime} \leq \infty$ and $s \in \mathbb{R}$, one has

$$
B_{p, 1}^{s}\left(\mathbb{R}^{n}\right) \hookrightarrow B_{p^{\prime}, 1}^{s-\frac{n}{p}+\frac{n}{p^{\prime}}}\left(\mathbb{R}^{n}\right)
$$

Let $s_{0} \neq s_{1} \in \mathbb{R}$ and $0 \leq p_{1}<p_{0} \leq \infty$. Let $f \in \mathcal{S}^{\prime}\left(\mathbb{R}^{n}\right)$ be such that $f \in$ $B_{p_{0}, 1}^{s_{0}}\left(\mathbb{R}^{n}\right) \cap B_{p_{1}, 1}^{s_{1}}\left(\mathbb{R}^{n}\right)$. Then, one gets

$$
f \in B_{p, q}^{s}\left(\mathbb{R}^{n}\right) \quad \text { for all } \frac{1}{p} \in\left[\frac{1}{p_{0}}, \frac{1}{p_{1}}\right] \text { and } s=\left(1-\theta_{p}\right) s_{0}+\theta_{p} s_{1}
$$

with $\theta_{p}=\frac{1 / p-1 / p_{0}}{1 / p_{1}-1 / p_{0}}$. This result is a direct consequence of the definition of the Besov spaces and of the Hölder inequality.

The proof of Proposition 1.2 relies on the following observations. 
(i) If there exists $t_{0} \geq 0$ such that $s_{f}\left(t_{0}\right)=-\infty$ then for all $t \geq t_{0}$, one has also $s_{f}(t)=-\infty$. It is a direct consequence of the embedding (1).

(ii) If there exists $t_{0} \geq 0$ such that $s_{f}\left(t_{0}\right)=+\infty$ then for all $t \leq t_{0}$, one has $s_{f}(t)=+\infty$. It also is a direct consequence of (1).

(iii) If $s_{f}(0)=+\infty$, then there exists at most one point $t_{0}$ such that $-\infty<s_{f}\left(t_{0}\right)<$ $+\infty$. Suppose indeed that there exist $t_{0}, t_{1} \geq 0$ with $t_{0}<t_{1}$ such that $s_{f}\left(t_{0}\right)=$ $s_{0}$ and $s_{f}\left(t_{1}\right)=s_{1}$ with $-\infty<s_{0}, s_{1}<+\infty$. Let $s \neq s_{1}-1 / 2 \in \mathbb{R}$ be such that

$$
s_{0}+1 \leq-\frac{\left(s-\left(s_{1}-1 / 2\right)\right)}{t_{1}} t_{0}+s .
$$

Then $f \in B_{\infty, 1}^{s}\left(\mathbb{R}^{n}\right)$ since $s_{f}(0)=\infty$ and $f \in B_{1 / t_{1}, 1}^{s_{1}-1 / 2}\left(\mathbb{R}^{n}\right)$ since $s_{f}\left(t_{1}\right)=s_{1}$. By the Hölder inequality $(2), f$ belongs to $B_{1 / t_{0}, 1}^{s_{0}+1}\left(\mathbb{R}^{n}\right)$, which is in contradiction with $s_{f}\left(t_{0}\right)=s_{0}$.

(iv) Suppose now that $-\infty<s_{f}(t)<\infty$ for all $t \in\left[0, t_{0}\right.$ [ with $0<t_{0} \leq \infty$. By the Hölder inequality $(2), s_{f}$ is continuous and concave on $\left[0, t_{0}[\right.$. Hence, $s_{f}$ is absolutely continuous and $s_{f}^{\prime}$ is well defined almost everywhere (see [4, chapter 7], for example). Moreover, by the embedding (1), we get that $s_{f}^{\prime} \leq n$ and that $s_{f}\left(t_{0}\right) \leq \lim _{t \rightarrow t_{0}^{-}} s_{f}(t)$.

Finally, $s_{f}$ satisfies one of the listed cases of Proposition 1.2.

We are now interested in the converse assertion, which is the object of Theorem 1.4. But let us first recall the definition of the singular support of a distribution.

Definition 1.3. Let $f \in \mathcal{S}^{\prime}\left(\mathbb{R}^{n}\right)$. The singular support of $f$ is the set

$$
\operatorname{sing} \operatorname{supp} f=\left\{x \in \mathbb{R}^{n} ; f \mid B(x, r) \notin \mathcal{C}^{\infty}\left(\mathbb{R}^{n}\right) \text { for any } r>0\right\} .
$$

Theorem 1.4. Let $\Gamma=\Gamma(t)$ be a function defined on $[0,+\infty[$ at values in $\overline{\mathbb{R}}$ such that $\Gamma$ satisfies one of the five cases of Proposition 1.2 (where $s_{f}$ is now replaced with $\Gamma$ ).

There exists a distribution $f \in \mathcal{S}^{\prime}\left(\mathbb{R}^{n}\right)$ such that $s_{f}=\Gamma$ and $\operatorname{sing} \operatorname{supp} f=\emptyset$.

\section{Proof of Theorem 1.4}

We will use the characterizations of Besov spaces with the Meyer wavelet basis. For that, let us introduce the quasi-Banach spaces $b_{p, q}^{s}$ for $s \in \mathbb{R}, 0<p \leq \infty, 0<q \leq \infty$.

For $j \in \mathbb{N}$, we define the subsets $G^{j}$ of $\{(F, M)\}^{n}$ by

$$
G^{0}=\{(F, M)\}^{n} \quad \text { and } \quad G^{j}=\{(F, M)\}^{n} \backslash\{(F, \ldots, F)\} \text { if } j \geq 1 .
$$


A sequence $c=\left\{c_{j, k}^{G}\right\}$ belongs to $b_{p, q}^{s}$ if and only if

$$
\|c\|_{b_{p, q}^{s}}=\left[\sum_{j \geq 0} \sum_{G \in G^{j}}\left|2^{j s} 2^{-j n / p}\left(\sum_{k}\left|c_{j, k}^{G}\right|^{p}\right)^{1 / p}\right|^{q}\right]^{1 / q}<\infty
$$

(usual modification for $p=\infty$ and/or $q=\infty$ ).

Let us fix $\psi_{F}$ a Meyer scaling function and $\psi_{M}$ the associated (mother) wavelet in one dimension. The wavelet basis of $L^{2}\left(\mathbb{R}^{n}\right)$, is the collection

$$
\left\{\psi_{j, k}^{G}=2^{n j / 2} \prod_{r=1}^{n} \psi_{G_{r}}\left(2^{j} x_{r}-k_{r}\right), \quad j \geq 0, k \in \mathbb{Z}^{n}, \quad G \in G^{j}\right\}
$$

with $x=\left(x_{1}, \ldots, x_{n}\right), G=\left(G_{1}, \ldots, G_{n}\right)$, and $k=\left(k_{1}, \ldots, k_{n}\right)$. We have the following result $([6$, chapter 3 , Theorem 3.12$])$.

Theorem 2.1. Let $0<p, q \leq \infty$ and $s \in \mathbb{R}$. Let $f \in \mathcal{S}^{\prime}\left(\mathbb{R}^{n}\right)$. Then $f \in B_{p, q}^{s}\left(\mathbb{R}^{n}\right)$ if, and only if, it can be represented as

$$
f=\sum_{j, k, G} c_{j, k}^{G} 2^{-j n / 2} \psi_{j, k}^{G}, \quad c \in b_{p, q}^{s},
$$

unconditional convergence being in $\mathcal{S}^{\prime}\left(\mathbb{R}^{n}\right)$ and locally in any $B_{p, q}^{\sigma}\left(\mathbb{R}^{n}\right)$ with $\sigma<s$. The representation is unique,

$$
c_{j, k}^{G}=2^{n j / 2}\left(f, \psi_{j, k}^{G}\right)
$$

and

$$
I: f \rightarrow\left\{2^{n j / 2}\left(f, \psi_{j, k}^{G}\right)\right\}
$$

is an isomorphic map of $B_{p, q}^{s}\left(\mathbb{R}^{n}\right)$ onto $b_{p, q}^{s}$. If in addition $p, q<\infty$ then $\left\{\psi_{j, k}^{G}\right\}$ is an unconditional basis of $B_{p, q}^{s}\left(\mathbb{R}^{n}\right)$.

Before studying the different cases of Theorem 1.4, let us give two lemmas which deal with the simpler examples where the Besov characteristic is a line or a piecewise linear function and which will be useful for the proof of this theorem.

Lemma 2.2. Let $a \in \mathbb{R}, a \leq n$, and $b \in \mathbb{R}$. There exists $f \in \mathcal{S}^{\prime}\left(\mathbb{R}^{n}\right)$ with $\operatorname{sing} \operatorname{supp} f=\emptyset$ such that

$$
s_{f}(t)=a t+b,
$$

and such that its wavelet coefficients $\left\{c_{j, k}^{G}\right\}=c$ are all non-negative and satisfy

$$
\|c\|_{b_{1 / t, 1}^{s}} \leq 1
$$

for all $t \geq 0$ and $s \leq s(t)$. 
Proof. Let $c$ be defined by

$$
c_{j, k}^{G}= \begin{cases}\frac{6}{\pi^{2}} \frac{1}{j^{2}} 2^{-j t b} & \text { if } j \geq 1, G=(M, \ldots, M) \text { and } k \in \widetilde{K_{j}}, \\ 0 & \text { otherwise, }\end{cases}
$$

where $\widetilde{K_{j}}$ is finite of cardinal $2^{j(n-a)}$ and is such that $|k| \geq 2^{j^{2}}$ if $k \in K_{j}$. An easy calculation shows that $\|c\|_{b_{1 / t, 1}^{s}}$ is finite if and only if $s \leq a t+b$ and in that case, we have $\|c\|_{b_{1 / t, 1}^{s}} \leq 1$. Noticing that the coefficients do not vanish for large $k$ only, we can show that $f$ defined by its wavelet expansion (3) belongs to $\mathcal{C}^{\infty}\left(\mathbb{R}^{n}\right)$ on any compact set and so sing supp $f=\emptyset$. Thus, $f$ satisfies the conclusion of Lemma 2.2.

Lemma 2.3. Let $\Gamma$ be a real-valued function on $[0, \infty[$. Assume that $\Gamma$ is continuous, concave, piecewise linear on the intervals $\left[t_{k}, t_{k+1}\left[, 1 \leq k \leq n\right.\right.$ with $0=t_{1}<t_{2}<$ $\cdots<t_{n}<t_{n+1}=+\infty$ and that $\Gamma^{\prime} \leq n$.

There exists $f \in \mathcal{S}^{\prime}\left(\mathbb{R}^{n}\right)$ with non-negative wavelet coefficients $\left\{c_{j, k}^{G}\right\}=c$, such that $\operatorname{sing} \operatorname{supp} f=\emptyset, s_{f}=\Gamma$, and

$$
\|c\|_{b_{1 / t, 1}^{s}} \leq 1
$$

Proof. Let $\Gamma$ be a function satisfying the assumptions of Lemma 2.3. There exist some reals $\left\{a_{p}, p \leq n\right\}$ with $a_{1} \geq a_{2} \geq \cdots \geq a_{n}$ and $\left\{b_{p}, p \leq n\right\}$ such that

$$
\Gamma(t)=a_{p} t+b_{p}
$$

on $\left[t_{p}, t_{p+1}\right]$. Thus, one has, for any $t \in[0, \infty[$,

$$
\Gamma(t)=\inf _{1 \leq p \leq n} a_{p} t+b_{p}
$$

For $1 \leq p \leq n$, let $f_{p}$ be a distribution according to Lemma 2.2 with non-negative wavelet coefficients $\left\{c_{j, k}^{G, p}\right\}=c^{p}$ such that sing supp $f_{p}=\emptyset, s_{f_{p}}(t)=a_{p} t+b_{p}$ for $t \in\left[0,+\infty\left[\right.\right.$ and $\left\|c^{p}\right\|_{b_{1 / t, 1}^{s}} \leq 1$ if $s \leq a_{p} t+b_{p}$. Then $f=\frac{1}{n} \sum_{p=1}^{n} f_{p}$ satisfies the conclusion of Lemma 2.3. Indeed, we clearly have $s_{f}(t) \geq \inf _{1 \leq p \leq n} s_{f_{p}}(t)=\Gamma(t)$ and, since the wavelet coefficients of $f_{p}, 1 \leq p \leq n$, are all non-negative, we get

$$
s_{f}(t)=\inf s_{f_{p}}(t)=\Gamma(t) .
$$

Moreover, the wavelet coefficients $\left\{c_{j, k}^{G}\right\}=c$, of $f$ satisfy $\|c\|_{b_{1 / t, 1}^{s}} \leq 1$ for all $t \geq 0$ and $s \leq s_{f}(t)$

Remark. The last argument of positivity of the wavelet coefficients can be replaced by observing that on each interval $] t_{p}, t_{p+1}\left[\right.$, one has $s_{f_{p}}<s_{f_{j}}$ for $j \neq p$. Then for all $t \in] t_{p}, t_{p+1}$ [ and for all $\varepsilon>0$ small enough, we have $f_{j} \in B_{1 / t, 1}^{s_{f_{p}}+\varepsilon}\left(\mathbb{R}^{n}\right)$ and $f_{p} \notin B_{1 / t, 1}^{s_{f_{p}}+\varepsilon}\left(\mathbb{R}^{n}\right)$. Hence $f \notin B_{1 / t, 1}^{s_{f_{p}}+\varepsilon}\left(\mathbb{R}^{n}\right)$ and $s_{f}(t)=s_{f_{p}}(t)$ on each interval $] t_{p}, t_{p+1}[$. Since $s_{f}$ is continuous by Proposition 1.2 , we get $s_{f}=\Gamma$ on $[0, \infty[$. 
Now, let $\Gamma$ be as in Theorem 1.4. Considering particular sequences $c$, we will construct distributions (given by their wavelet expansions) which have $\Gamma$ as its Besov characteristic.

First case. $\quad \Gamma \equiv+\infty$ on $[0, \infty[$.

As it has already been said, any function of the Schwartz class gives the result.

Second case and fourth case. $\Gamma \equiv-\infty$ on $] 0, \infty\left[\right.$ or there exists $t_{0}>0$ such that

$$
s_{f}(t)= \begin{cases}+\infty & \text { if } t \in\left[0, t_{0}[\right. \\ s_{0} & \text { if } t=t_{0}, s_{0} \in \overline{\mathbb{R}} \\ -\infty & \text { if } t \in] t_{0},+\infty[\end{cases}
$$

Let us consider the sequence $c$ given by

$$
c_{j, k}^{G}= \begin{cases}\frac{1}{j^{2}} 2^{-j^{2} n t_{0}} 2^{j n t_{0}} 2^{-j s_{0}} & \text { if } j \geq 2, G=(M, \ldots, M), \text { and } k \in K_{j}, \\ 0 & \text { otherwise, }\end{cases}
$$

where $K_{j}$ is such that $|k| \geq 2^{j^{2}}$ if $k \in K_{j}$ and $\operatorname{Card} K_{j}=2^{n j^{2}}$. One has

$$
\|c\|_{b_{1 / t, 1}^{s}}=\sum_{j \geq 2} \frac{1}{j^{2}} 2^{j\left(s-s_{0}\right)} 2^{-j n\left(t-t_{0}\right)} 2^{-j^{2} n\left(t_{0}-t\right)} .
$$

Hence, if $t<t_{0}$, then $\|c\|_{b_{1 / t, 1}^{s}}$ is finite for all $s \in \mathbb{R}$. If $t=t_{0},\|c\|_{b_{1 / t, 1}^{s}}$ is finite for all $s \leq s_{0}$. If $t>t_{0},\|c\|_{b_{1 / t, 1}^{s}}=\infty$ for all $s \in \mathbb{R}$.

We define $f \in \mathcal{S}^{\prime}\left(\mathbb{R}^{n}\right)$ by

$$
f=\sum_{j, k, G} c_{j, k}^{G} \psi_{j, k}^{G}
$$

Then, $f$ belongs to $B_{1 / t, 1}^{s}\left(\mathbb{R}^{n}\right)$ if and only if $\|c\|_{b_{1 / t, 1}^{s}}<\infty$ and we obtain the second case (putting $t_{0}=0$ ) and the fourth case with $s_{0} \in \mathbb{R}$. Moreover, the singular support of $f$ is empty since $f$ is in $\mathcal{C}^{\infty}\left(\mathbb{R}^{n}\right)$ on every compact subset of $\mathbb{R}^{n}$. Indeed, let $R>0$, $x \in B(0, R)$ and $j_{0} \geq 2$ be such that $2^{j_{0}^{2}} \geq 2^{j_{0}+1} R$. Then, we have, for $\alpha \in \mathbb{N}^{n}$ and with $G^{(0)}=(M, \ldots, M)$,

$$
\begin{aligned}
\sum_{j, G, k}\left|c_{j, k}^{G} \partial^{\alpha} \psi_{j, k}^{G}(x)\right| \leq & \sum_{j \leq j_{0}} \sum_{k \in K_{j}}\left|c_{j, k}^{G^{(0)}} 2^{j|\alpha|} \partial^{\alpha} \psi^{G^{(0)}}\left(2^{j} x-k\right)\right| \\
& +\sum_{j \geq j_{0}} \sum_{k \in K_{j}}\left|c_{j, k}^{G^{(0)}} 2^{j|\alpha|} \partial^{\alpha} \psi^{G^{(0)}}\left(2^{j} x-k\right)\right| \\
\leq & C\left(j_{0}\right)\left\|\partial^{\alpha} \psi^{G^{(0)}}\right\|_{\infty} \\
& +\sum_{j \geq j_{0}} \sum_{k \in K_{j}}\left|c_{j, k}^{G^{(0)}} 2^{j|\alpha|} \partial^{\alpha} \psi^{G^{(0)}}\left(2^{j} x-k\right)\right|
\end{aligned}
$$


Since $\psi^{G^{(0)}}$ belongs to the Schwartz class and $\left|2^{j} x-k\right| \simeq|k|$ for $|k| \geq 2^{j^{2}}, j \geq j_{0}$, and $x \in B(0, R)$, we have the following estimate for all $M \in \mathbb{N}$ :

$$
\left|\partial^{\alpha} \psi^{G^{(0)}}\left(2^{j} x-k\right)\right| \leq \frac{C}{\left(1+\left|2^{j} x-k\right|\right)^{M}} \leq 2^{-j^{2} M} .
$$

Taking $M>n-n t_{0}$, it follows that

$$
\sum_{j, G, k}\left|c_{j, k}^{G} \partial^{\alpha} \psi_{j, k}^{G}(x)\right| \leq C(R) .
$$

Thus, the $\alpha$-th derivative series is normally convergent on each compact set and $\operatorname{sing} \operatorname{supp} f=\emptyset$.

Considering the sequence

$$
c_{j, k}^{G}= \begin{cases}2^{-j^{2} n t_{0}} 2^{j n t_{0}} 2^{-j^{3 / 2}} & \text { if } j \geq 2, G=(M, \ldots, M), \text { and } k \in K_{j}, \\ 0 & \text { otherwise }\end{cases}
$$

in the wavelet expansion (3), we obtain the second and fourth cases with $s_{0}=\infty$. With the sequence

$$
c_{j, k}^{G}= \begin{cases}2^{-j^{2} n t_{0}} 2^{j n t_{0}} 2^{j^{3 / 2}} & \text { if } j \geq 2, G=(M, \ldots, M), \text { and } k \in K_{j}, \\ 0 & \text { otherwise }\end{cases}
$$

the result follows for $s_{0}=-\infty$.

We are now interested in the case where the function $\Gamma$ is concave and continuous on $\left[0, t_{0}\left[\right.\right.$ with $0<t_{0} \leq \infty$. The idea is to approximate $\Gamma$ with piecewise linear functions.

Fifth case. Let $\Gamma$ be a concave and continuous function on $\left[0, t_{0}\left[=I\left(t_{0}>0\right)\right.\right.$ with $\Gamma^{\prime} \leq n,-\infty \leq \Gamma\left(t_{0}\right) \leq \lim _{t \rightarrow t_{0}^{-}} \Gamma(t)$ and $\Gamma \equiv-\infty$ on $] t_{0},+\infty[$.

Let $l \geq 0$. There exists $M_{l} \geq 0$ such that $\left|\Gamma^{\prime}\right| \leq M_{l}$ a.e. on $\left[0, t_{0}-2^{-l}\right]=I_{l}$. Then, there exists a piecewise linear function $\Gamma_{l} \geq \Gamma$ such that, for all $t \in I_{l}$,

$$
\left|\Gamma_{l}(t)-\Gamma(t)\right| \leq 2^{-l}
$$

(see figure 1).

By Lemma 2.3, we can construct a distribution $f_{l}$ with non-negative wavelet coefficients $\left\{c_{j, k}^{G, l}\right\}=c^{l}$ such that $\operatorname{sing} \operatorname{supp} f_{l}=\emptyset, s_{f_{l}}=\Gamma_{l}$, and $\left\|c^{l}\right\|_{b_{1 / t, 1}^{s}} \leq 1$ for all $t \geq 0$ and $s \leq \Gamma_{l}(t)$. Let us define $f \in \mathcal{S}^{\prime}\left(\mathbb{R}^{n}\right)$ by

$$
f=\sum_{l \geq 0} 2^{-l} f_{l}
$$




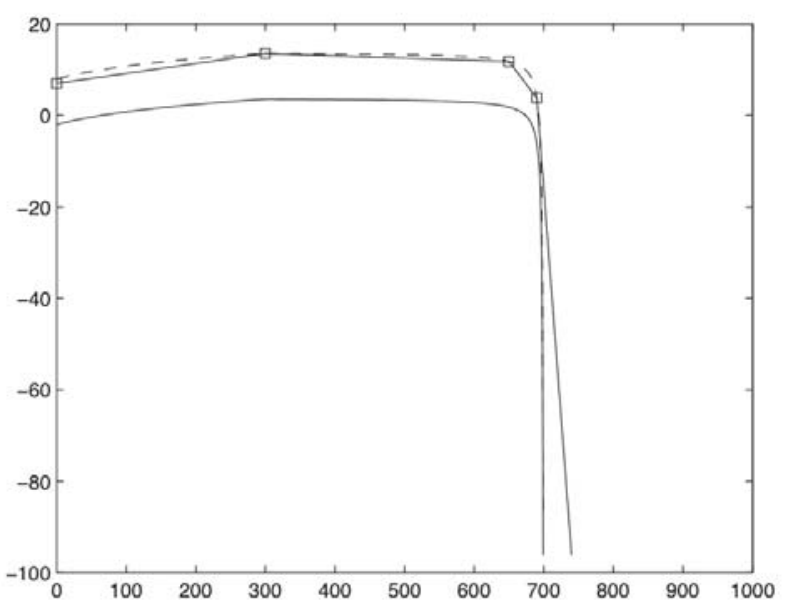

Figure 1 - Approximation of $\Gamma$ by $\Gamma_{l}$ on $\left[0, t_{0}-2^{-l}\right]$

and denote by $c=\left\{c_{j, k}^{G}\right\}$ its wavelet coefficients. They are given, for $j \geq 0, G \in G^{j}$, and $k \in \mathbb{Z}^{n}$, by

$$
c_{j, k}^{G}=\sum_{l \geq 0} 2^{-l} c_{j, k}^{G, l} .
$$

Clearly, the singular support of $f$ is empty. Let us now determine its Besov characteristic. If $t \geq 0$ and $s<\Gamma(t)$, it comes out that

$$
\|c\|_{b_{1 / t, 1}^{s}} \leq \sum_{l \geq 0} 2^{-l}\left\|c^{l}\right\|_{b_{1 / t, 1}^{s}} \leq \sum_{l} 2^{-l}<+\infty .
$$

Hence, $f$ belongs to $B_{1 / t, 1}^{s}\left(\mathbb{R}^{n}\right)$ and $s_{f}(t) \geq \Gamma(t)$. If $t \in I$ and $s>\Gamma(t)$, there exists $l_{0}$ such that $s_{f_{0}}(t)=\Gamma_{l_{0}}(t)<s$. Since all the coefficients are non-negative, one has $c_{j, k}^{G} \geq c_{j, k}^{G, l_{0}} \geq 0$ for all $j \in \mathbb{N}, G \in G^{j}$, and $k \in \mathbb{Z}^{n}$. It follows that

$$
\|c\|_{b_{1 / t, 1}^{s}} \geq\left\|c^{l_{0}}\right\|_{b_{1 / t, 1}^{s}}=\infty
$$

Finally, we get $s_{f}=\Gamma$ on $I$ and $s_{f} \geq \Gamma$ on $[0, \infty[$.

Taking a distribution $g \in \mathcal{S}^{\prime}\left(\mathbb{R}^{n}\right)$ with non-negative wavelets coefficients and such that $g \equiv+\infty$ on $\left[0, t_{0}\left[, g\left(t_{0}\right)=\Gamma\left(t_{0}\right)\right.\right.$ and $g \equiv-\infty$ on $] t_{0}, \infty\left[\right.$, we obtain that $s_{f+g}=\Gamma$.

Third case. $\Gamma$ is continuous, concave and $\Gamma^{\prime} \leq n$ on $[0, \infty[$.

The proof of the fifth case can easily be adapted to obtain this case, replacing $I=\left[0, t_{0}\left[\right.\right.$ with $\left[0, \infty\left[\right.\right.$ and $I_{l}=\left[0, t_{0}-2^{-l}\right]$ with $[0, l]$ (see figure 2 ). 


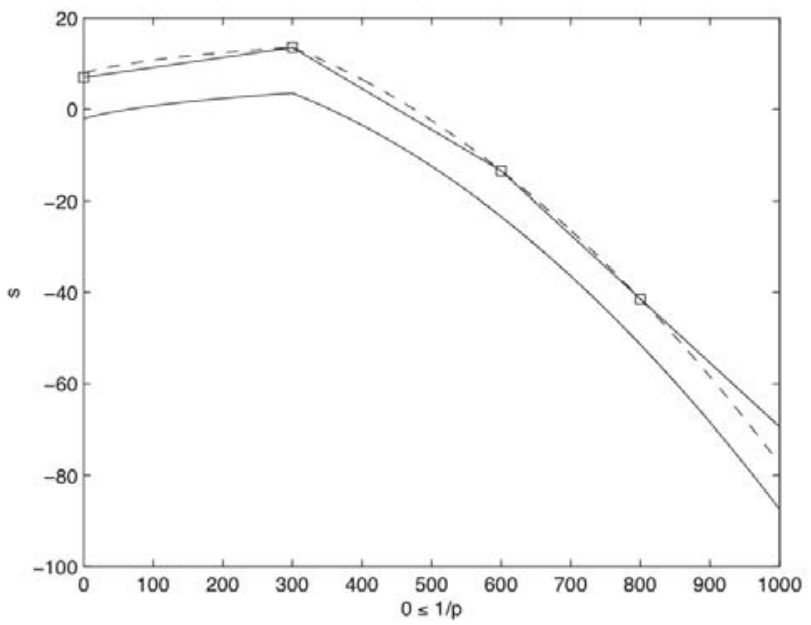

Figure 2 - Approximation of $\Gamma$ by $\Gamma_{l}$ on $[0, l]$.

Remark. For the second and fourth cases, if $t_{0}>0$ (or $t_{0}=0$ and $s_{0}=+\infty$ ), the fact that sing $\operatorname{supp} f=\emptyset$ follows immediately from the fact that $f \in B_{\infty, 1}^{\infty}\left(\mathbb{R}^{n}\right)$.

\section{Generalization to the weighted and to the anisotropic cases}

\subsection{Weighted Besov characteristic}

We restrict the study to a class of weights for which the result of Proposition 1.2 and Theorem 1.4 can be immediately generalized to the weighted Besov spaces.

For $\alpha \in \mathbb{R}$ and $x \in \mathbb{R}^{n}$, we define the weight $w_{\alpha}$ by

$$
w_{\alpha}(x)=\left(1+|x|^{2}\right)^{|\alpha| / 2} .
$$

Definition 3.1. Let $s, \alpha \in \mathbb{R}$ and $0<p, q \leq \infty$. The weighted Besov space $B_{p, q}^{s}\left(\mathbb{R}^{n}, \alpha\right)$ is the collection of all distributions $f \in \mathcal{S}^{\prime}\left(\mathbb{R}^{n}\right)$ such that $f w_{\alpha} \in B_{p, q}^{s}\left(\mathbb{R}^{n}\right)$. We put

$$
\|f\|_{B_{p, q}^{s}\left(\mathbb{R}^{n}, \alpha\right)}=\left\|f w_{\alpha}\right\|_{B_{p, q}^{s}\left(\mathbb{R}^{n}\right)} .
$$

Definition 3.2. Let $g \in \mathcal{S}^{\prime}\left(\mathbb{R}^{n}\right)$. The weighted Besov characteristic of $g$ is the function $s_{g}^{\alpha}$ defined for $t \in \mathbb{R}^{+}$and at values in $\overline{\mathbb{R}}$ by

$$
s_{g}^{\alpha}(t)=\sup \left\{s \in \mathbb{R} ; g \in B_{1 / t, 1}^{s}\left(\mathbb{R}^{n}, \alpha\right)\right\} .
$$

Proposition 3.3. $\quad$ (i) Let $g \in \mathcal{S}^{\prime}\left(\mathbb{R}^{n}\right)$. Then its weighted Besov characteristic $s_{g}^{\alpha}$ satisfies one of the five cases of Proposition 1.2 (replacing $s_{f}$ with $s_{g}^{\alpha}$ ). 
(ii) Let $\Gamma=\Gamma(t)$ be a function defined on $[0, \infty[$ at values in $\overline{\mathbb{R}}$ such that $\Gamma$ satisfies one of the five cases of Proposition 1.2 (where $s_{f}$ is now replaced by $\Gamma$ ). There exists a distribution $g \in \mathcal{S}^{\prime}\left(\mathbb{R}^{n}\right)$ such that $s_{g}^{\alpha}=\Gamma$.

Proof. The proof is a direct consequence of the result in the unweighted case. Indeed, one has, by definition

$$
s_{g}^{\alpha}(t)=\sup \left\{s \in \mathbb{R} ; g w_{\alpha} \in B_{1 / t, 1}^{s}\left(\mathbb{R}^{n}\right)\right\},
$$

which means that $s_{g}^{\alpha}$ is the Besov characteristic of $g w_{\alpha}$. Hence $s_{g}^{\alpha}$ satisfies one of the five cases of Proposition 1.2.

Conversely, let $\Gamma$ satisfy one of the five cases of Proposition 1.2. Then, by Theorem 1.4, there exists $f \in \mathcal{C}_{\text {loc }}^{\infty}\left(\mathbb{R}^{n}\right)$ such that $s_{f}=\Gamma$. But $g=\left(1+|x|^{2}\right)^{-\alpha / 2} f$ belongs to $L_{\text {loc }}^{1}\left(\mathbb{R}^{n}\right) \subset \mathcal{S}^{\prime}\left(\mathbb{R}^{n}\right)$ and $s_{g}^{\alpha}=s_{f}=\Gamma$.

\subsection{Anisotropic Besov characteristic}

By the transference method proposed by Triebel in [6, chapter 3 , section 5]), it is possible to extend the results of sections 1 and 2 to the anisotropic case. Let us first recall the definitions of anisotropic Besov spaces and characteristic.

An $n$-tuple $\alpha \in \mathbb{R}^{n}$ is called an anisotropy in $\mathbb{R}^{n}$ if and only if

$$
0<\alpha_{1} \leq \cdots \leq \alpha_{n}<\infty \text { and } \sum_{j=1}^{n} \alpha_{j}=n
$$

For $t>0, r \in \mathbb{R}$ and $x \in \mathbb{R}^{n}$ we put

$$
t^{\alpha} x=\left(t^{\alpha_{1}} x_{1}, \ldots, t^{\alpha_{n}} x_{n}\right) \text { and } t^{r \alpha}=\left(t^{r}\right)^{\alpha} \text {. }
$$

We define a smooth anisotropic resolution of unity $\varphi_{j}^{\alpha}$ in the following way. The function $\varphi_{0}^{\alpha} \in \mathcal{S}\left(\mathbb{R}^{n}\right)$ satisfies

$$
\varphi_{0}^{\alpha}(\xi)=1 \quad \text { if } \sup _{l}\left|\xi_{l}\right| \leq 1 \quad \text { and } \quad \varphi_{0}^{\alpha}(\xi)=0 \quad \text { if } \sup _{l} 2^{-\alpha_{l}}\left|\xi_{l}\right| \geq 1 \text {, }
$$

where $\xi=\left(\xi_{1}, \ldots, \xi_{n}\right) \in \mathbb{R}^{n}$. Let

$$
\varphi_{j}^{\alpha}(\xi)=\varphi_{0}^{\alpha}\left(2^{-j \alpha} \xi\right)-\varphi_{0}^{\alpha}\left(2^{-(j-1) \alpha} \xi\right), \quad j \geq 1, \quad \xi \in \mathbb{R}^{n} .
$$

One has

$$
\begin{gathered}
\sum_{j=0}^{\infty} \varphi_{j}^{\alpha} \equiv 1 \quad \text { on } \mathbb{R}^{n}, \\
\operatorname{supp} \varphi_{0}^{\alpha} \subset R_{1}^{\alpha} \quad \text { and } \operatorname{supp} \varphi_{k}^{\alpha} \subset R_{k+1}^{\alpha} \backslash R_{k-1}^{\alpha}, \quad k \geq 1,
\end{gathered}
$$


where the sets $R_{j}^{\alpha}$ are the rectangles

$$
R_{j}^{\alpha}=\left\{x \in \mathbb{R}^{n} ;\left|x_{l}\right| \leq 2^{j \alpha_{l}}\right\}, \quad j \geq 0 .
$$

We define the operators $\Delta_{j}^{\alpha}$ on $\mathcal{S}^{\prime}\left(\mathbb{R}^{n}\right)$ by

$$
\widehat{\Delta_{j, \alpha}(f)}=\varphi_{j}^{\alpha} \hat{f}
$$

For $0<p \leq \infty, 0<q \leq \infty$, and $s \in \mathbb{R}$, the anisotropic Besov space $B_{p, q}^{s, \alpha}\left(\mathbb{R}^{n}\right)$ is defined by

$$
\left\{f \in \mathcal{S}^{\prime}\left(\mathbb{R}^{n}\right) ;\|f\|_{B_{p, q}^{s, \alpha}}=\left(\sum_{j=0}^{\infty} 2^{j s q}\left\|\Delta_{j}^{\alpha}(f)\right\|_{L^{p}}^{q}\right)^{1 / q}<\infty\right\}
$$

(usual modification if $q=\infty$ ).

Remark. The definition of $B_{p, q}^{s, \alpha}\left(\mathbb{R}^{n}\right)$ does not depend on the choice of the resolution $\left\{\varphi_{j}^{\alpha}\right\}$. These spaces are quasi-Banach spaces and are Banach spaces if $p \geq 1$ and $q \geq 1$ (see [5, section 2.3.3]).

Remark. For $\alpha=(1, \ldots, 1)$, we obtain $B_{p, q}^{s, \alpha}\left(\mathbb{R}^{n}\right)=B_{p, q}^{s}\left(\mathbb{R}^{n}\right)$.

Lemma 3.4. Let $\alpha$ be an anisotropy. Let $0<p, q<\infty$ and $s_{0}, s_{1} \in \mathbb{R}, s_{0}>s_{1}$. Then $B_{p, q}^{s_{0}, \alpha}\left(\mathbb{R}^{n}\right) \subset B_{p, q}^{s_{1}, \alpha}\left(\mathbb{R}^{n}\right)$.

Definition 3.5. Let $f \in \mathcal{S}^{\prime}\left(\mathbb{R}^{n}\right)$. The anisotropic Besov characteristic of $f$ is the function $s_{f}^{\alpha}$ defined for $t \in[0, \infty[$ at values in $\overline{\mathbb{R}}$ by

$$
s_{f}^{\alpha}(t)=\sup \left\{s \in \mathbb{R} ; f \in B_{1 / t, 1}^{s, \alpha}\left(\mathbb{R}^{n}\right)\right\} .
$$

In the case of compactly supported distributions, Triebel has shown the following result in $[6$, chapter 5 , section 3.4$]$.

Theorem 3.6. Let $\alpha$ be an anisotropy.

(i) Let $f \in \mathcal{S}^{\prime}\left(\mathbb{R}^{n}\right)$ be not in $\mathcal{C}^{\infty}\left(\mathbb{R}^{n}\right)$ and let supp $f$ be compact. Then $s_{f}^{\alpha}$ is an increasing concave function of slope smaller than or equal to $n$.

(ii) For any real increasing concave function $\Gamma$ on $[0, \infty$ [ of slope smaller than or equal to $n$ there is a compactly supported distribution $f \in \mathcal{S}^{\prime}\left(\mathbb{R}^{n}\right)$ such that $s_{f}^{\alpha}=\Gamma$.

Without any assumption on the compactness of the support, we obtain the following result.

Theorem 3.7. Let $\alpha$ be an anisotropy. 
(i) Let $f \in \mathcal{S}^{\prime}\left(\mathbb{R}^{n}\right)$. Then its anisotropic Besov characteristic $s_{f}^{\alpha}$ satisfies one of the five cases of Proposition 1.2 (replacing $s_{f}$ with $s_{f}^{\alpha}$ ).

(ii) Let $\Gamma=\Gamma(t)$ be a function defined on $[0, \infty[$ at values in $\overline{\mathbb{R}}$ such that $\Gamma$ satisfies one of the five cases of Proposition 1.2 (where $s_{f}$ is now replaced by $\Gamma$ ). There exists a distribution $f \in \mathcal{S}^{\prime}\left(\mathbb{R}^{n}\right)$ such that $s_{f}^{\alpha}=\Gamma$.

The proof follows exactly the same arguments of the proof of Theorem 3.6, which is based on the transference method (section 5.3 of [6]). We will indeed show that we can transfer the result from the isotropic case to the anisotropic case, via the Meyer representation of the spaces. Let us first recall the construction of the anisotropic Meyer wavelets.

We fix an anisotropy $\alpha$. Again $\psi_{F}$ denotes the Meyer scaling function in one dimension and $\psi_{M}$ the associated wavelet. For an integer $n \geq 2$, we denote by $I^{j, \alpha}$ the sets

$$
I^{j, \alpha} \subset\{F, M\}^{n} \times \mathbb{N}_{0}^{n}, \quad j \in \mathbb{N}_{0} .
$$

We put $\{F, M\}^{n *}=\{F, M\}^{n} \backslash\{(F, \ldots, F)\}$. Then, by definition, one has $I^{0, \alpha}=$ $\{((F, \ldots, F),(0, \ldots, 0))\}$. If $j \geq 1$, by definition $I^{j, \alpha}$ is the collection of all elements $(G, k)$ with $G \in\{F, M\}^{n *}$ and $k \in \mathbb{N}_{0}^{n}$ such that

$$
k_{r}=\left[(j-1) \alpha_{r}\right] \quad \text { if } G_{r}=F
$$

and

$$
\left[(j-1) \alpha_{r}\right] \leq k_{r}<\left[j \alpha_{r}\right] \quad \text { if } G_{r}=M,
$$

for $r=1, \ldots, n$ and $k=\left(k_{1}, \ldots, k_{n}\right)$.

Remark. Since $\alpha_{n} \geq 1$, in all the anisotropic cases, the set $I^{j, \alpha}$ with $j \in \mathbb{N}$ satisfies

$$
1 \leq \operatorname{Card} I^{j, \alpha} \leq\left(2^{n}-1\right) \prod_{r=1}^{n}\left(1+\left[j \alpha_{r}\right]-\left[(j-1) \alpha_{r}\right]\right) \leq\left(2^{n}-1\right) \prod_{r=1}^{n}\left(2+\alpha_{r}\right) .
$$

The anisotropic Meyer wavelet basis is then the collection

$$
\left\{\psi_{j, m}^{(G, k), \alpha}(x)=2^{|k| / 2} \prod_{r=1}^{n} \psi_{G_{r}}\left(2^{k_{r}} x_{r}-m_{r}\right), \quad j \in \mathbb{N}_{0}, \quad(G, k) \in I_{j, \alpha}, m \in \mathbb{Z}^{n}\right\}
$$

where $|k|=k_{1}+k_{2}+\cdots+k_{n}$. Let us define the anisotropic quasi-Banach spaces $b_{p, q}^{s, \alpha}$ for $s \in \mathbb{R}, 0<p \leq \infty$, and $0<q \leq \infty$. For $c=\left\{c_{j, m}^{(G, k)} \in \mathbb{C}, j \in \mathbb{N}_{0},(G, k) \in I^{j, \alpha}\right.$, $\left.m \in \mathbb{Z}^{n}\right\}$, we put

$$
\|c\|_{b_{p, q}^{s, \alpha}}=\left(\sum_{j=0}^{\infty} \sum_{(G, k) \in I^{j, \alpha}} 2^{j(s-n / p) q}\left(\sum_{m \in \mathbb{Z}^{n}}\left|c_{j, m}^{(G, k)}\right|^{p}\right)^{q / p}\right)^{1 / q}
$$

(usual modification for $p=\infty$ and/or $q=\infty$ ). Then

$$
b_{p, q}^{s, \alpha}=\left\{c ;\|c\|_{b_{p, q}^{s, \alpha}}<\infty\right\} .
$$


Theorem 3.8 ([6, chapter 5, section 2]). Let $\alpha$ be an anisotropy and $\psi_{j, m}^{(G, k), \alpha}$ the anisotropic Meyer wavelets. Let $0<p, q \leq \infty$ and $s \in \mathbb{R}$. Then $f$ belongs to $B_{p, q}^{s, \alpha}\left(\mathbb{R}^{n}\right)$ if, and only if, it can be represented as

$$
f=\sum_{j,(G, k), m} c_{j, m}^{(G, k)} 2^{-|k| / 2} \psi_{j, m}^{(G, k), \alpha}, \quad c \in b_{p, q}^{s, \alpha},
$$

unconditional convergence being in $\mathcal{S}^{\prime}\left(\mathbb{R}^{n}\right)$ and locally in any $B_{p, q}^{\sigma, \alpha}\left(\mathbb{R}^{n}\right)$ with $\sigma<s$. The representation (4) is unique,

$$
c_{j, m}^{(G, k)}=2^{|k| / 2}\left(f, \psi_{j, m}^{(G, k), \alpha}\right)
$$

and

$$
I: f \rightarrow\left\{2^{|k| / 2}\left(f, \psi_{j, m}^{(G, k), \alpha}\right)\right\}
$$

is an isomorphic map of $B_{p, q}^{s, \alpha}$ onto $b_{p, q}^{s, \alpha}$. If in addition $p<\infty$ and $q<\infty$, then $\left\{\psi_{j, m}^{(G, k), \alpha}\right\}$ is an unconditional basis in $B_{p, q}^{s, \alpha}\left(\mathbb{R}^{n}\right)$.

For more details and for proofs on the anisotropic Meyer and Daubechies wavelets and the anisotropic multiresolution, we refer again to [6, chapter 5, section 2].

Proof (of Theorem 3.7). Via the universal Meyer representation, we can transfer the assertions of Proposition 1.2 and of Theorem 1.4 to the anisotropic case. Let $f \in \mathcal{S}^{\prime}\left(\mathbb{R}^{n}\right)$ be given by its wavelet expansion

$$
f=\sum_{j \in \mathbb{N}} \sum_{G \in G^{j}} \sum_{k \in \mathbb{Z}^{n}} c_{j, k}^{G} 2^{-j n / 2} \psi_{j, k}^{G} .
$$

Let $g \in \mathcal{S}^{\prime}\left(\mathbb{R}^{n}\right)$ be given by

$$
g=\sum_{j=0}^{\infty} \sum_{(G, k) \in I_{j, \alpha}} \sum_{m \in \mathbb{Z}^{n}} \lambda_{j, m}^{(G, k)} 2^{-|k| / 2} \psi_{j, m}^{(G, k), \alpha}
$$

where the coefficients $\lambda_{j, m}^{(G, k)}$ are either 0 or coincide for fixed $j \in \mathbb{N}_{0}$ one-to-one with the coefficients $c_{j, k}^{G}$. Then, we have $s_{g}^{\alpha}=s_{f}$. Conversely, for $g \in \mathcal{S}^{\prime}\left(\mathbb{R}^{n}\right)$, we can construct a distribution $f$ such that $s_{f}=s_{g}^{\alpha}$ and Theorem 3.7 follows.

\section{References}

[1] D. E. Edmunds and H. Triebel, Function spaces, entropy numbers, differential operators, Cambridge Tracts in Mathematics, vol. 120, Cambridge University Press, Cambridge, 1996.

[2] S. Jaffard, On the Frisch-Parisi conjecture, J. Math. Pures Appl. (9) 79 (2000), no. 6, 525-552.

[3] S. Jaffard, Wavelet expansions, function spaces and multifractal analysis, Twentieth Century Harmonic Analysis: A Celebration (Il Ciocco, 2000), NATO Sci. Ser. II Math. Phys. Chem., vol. 33, Kluwer Acad. Publ., Dordrecht, 2001, pp. 127-144. 
[4] W. Rudin, Real and complex analysis, 3rd ed., McGraw-Hill Book Co., New York, 1987.

[5] H. Triebel, Theory of function spaces, Monographs in Mathematics, vol. 78, Birkhäuser Verlag, Basel, 1983.

[6] _ Theory of function spaces, III, Monographs in Mathematics, vol. 100, Birkhäuser Verlag, Basel, 2006. 